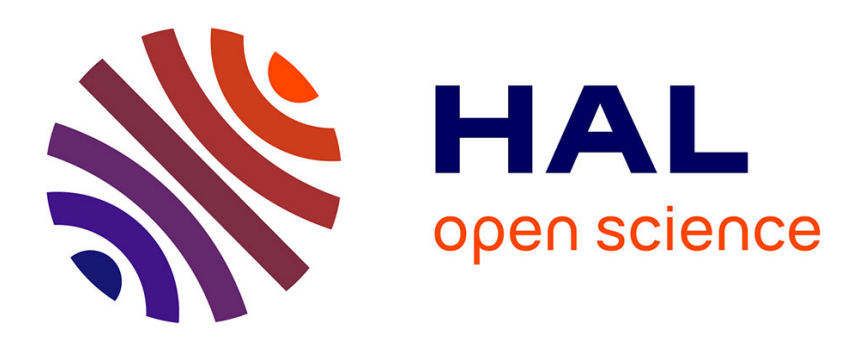

\title{
Light interactions with supracrystals either deposited on a substrate or dispersed in water \\ Marie Paule Pileni
}

\section{To cite this version:}

Marie Paule Pileni. Light interactions with supracrystals either deposited on a substrate or dispersed in water. Inorganic Chemistry Frontiers, 2020, 7 (20), pp.3796 - 3804. 10.1039/d0qi00353k . hal03003247

\section{HAL Id: hal-03003247 \\ https://hal.sorbonne-universite.fr/hal-03003247}

Submitted on 13 Nov 2020

HAL is a multi-disciplinary open access archive for the deposit and dissemination of scientific research documents, whether they are published or not. The documents may come from teaching and research institutions in France or abroad, or from public or private research centers.
L'archive ouverte pluridisciplinaire HAL, est destinée au dépôt et à la diffusion de documents scientifiques de niveau recherche, publiés ou non, émanant des établissements d'enseignement et de recherche français ou étrangers, des laboratoires publics ou privés. 


\section{Light interactions with supracrystals either deposited on a substrate or dispersed in water}

Marie Paule Pileni

Nanocrystals with low size distribution are able to self-assemble into a 3D crystalline structure called colloidal crystals or super/supracrystals. A rather large number of supracrystal specific properties have been achieved showing promising potential applications. Here, we compared intrinsic pro- perties induced by light interacting with fcc supracrystals of hydrophobic metal nanocrystals either deposited on a substrate or dispersed in aqueous solution. We first describe the formation of a dried supracrystal film grown via a heterogeneous process with cracks formed due to the shrinking of the film caused by restriction of its adhesion on the surface. We also describe the method to fabricate hydrophobic supracrystals dispersed in aqueous solution. The optical properties of the thick dried supracrystal film are determined from the wetting layers formed at the bottom of the cracks whereas, for water dispersed suprastructures, both the collective optical photonic mode and absorption of dispersed nanocrystals used as build blocks are observed. Ag nanocrystals used as building blocks in a dried supracrystal film vibrate coherently as atoms in a nanocrystal. However, it is impossible to determine the oscillation period of the whole assembly. Conversely from a dynamic study, the breathing period of the assemblies dispersed in aqueous solution is found to be around 300 ps. Whatever experimental conditions, nanocrystals exposed to light breath coherently in a supracrystal. In aqueous solution, supracrystals behave as nanoheaters.

Received 24th March 2020, Accepted 19th May 2020

DOI: $10.1039 / \mathrm{d} 0 \mathrm{qi} 00353 \mathrm{k}$

rsc.li/frontiers-inorganic

\section{Introduction}

Spontaneous self-assemblies are universally studied with building blocks from either atoms or nanomaterials. Similarly, to the crystalline growth at the atomic level, assemblies of colloidal crystals are as old as the Universe. Examples are assemblies of silicate particles result in opals which are characterized by their well-known specific reflectivity related to the size of segregated ordered particles. ${ }^{1}$ The changes of their optical properties are due to particles ordered at the mesoscopic scale revealing the emergence of specific properties.

Artificial colloidal crystals, also called supracrystals, of spherical single component spherical inorganic nanocrystals, coated with hydrophobic alkyl chains generate various crystalline structures (face centered cubic, $f c c$, hexagonal close packed, $h c p$ and body centered cubic, $b c c$.) Such crystalline structures are determined by minimizing the system's free energy F = U - TS. Note that because of the thermal energy is a constant, entropy, 
$\mathrm{S}$, is the key parameter. ${ }^{2-18}$ By mixing nanocrystals having two different well-defined sizes, they self-assemble to form crystalline structures of different types such as $\mathrm{NaCl}, \mathrm{AlB}_{2}-\mathrm{NaZn}_{13}$ etc. ${ }^{\mathbf{2 0 - 2 2}}$

Supracrystals are a new generation of advanced materials exhibiting unique collective and intrinsic chemical (stability, nano-kinkerdall) and physical (optical, magnetic, mechanical, vibrational, crystal growth processes, etc.) properties, which differ from those of disordered aggregates or of isolated nanocrystals or of the bulk phase $\mathbf{6 , 8}, \mathbf{9}, \mathbf{1 1}, \mathbf{1 4}, \mathbf{2 1 - 2 9}$. These supracrystal properties make possible the exploration of a large variety of potential applications.

Here we compared intrinsic properties induced by light interacting with fcc supracrystals of hydrophobic nanocrystals are either deposited on a substrate or dispersed in aqueous solution.

\section{Supracrystals of hydrophobic nanocrystals either deposited on a substrate or dispersed in aqueous} solution.

In order to self-assemble coated nanocrystals in crystalline structures, we need to produce nanocrystals having a size distribution less than $10 \%$. This compulsory condition is not unique. Several other parameters must be taken into account such as the nanocrystal coating agent used to keep the integrity, temperature, solvent vapor pressure, trace of water molecules etc..... All the parameters are related to the interactions between alkyl chains, solvent/ alkyl chain and nanocrystals.

Over these two decades, a rather large number of methods have been developed to produce nanocrystals selfassembled in crystalline structure such as fcc, hcp, bcc as atoms in atomic crystals. Such assemblies are called supracrystals. Actually, two growth processes exist: The first one is a heterogeneous growth with formation of nanocrystals assemble layer by layer ((Fig.1a and b). The second one is a homogeneous growth process with formation of shaped supracrystals in solution. In some case, the supracrystal shapes are similar to those of single atomic crystals. On Figures 1c and 1d, it shown a supracrystal with a five-fold symmetry as icosahedral terminated by (111) planes corresponding to multiply twinned crystals.

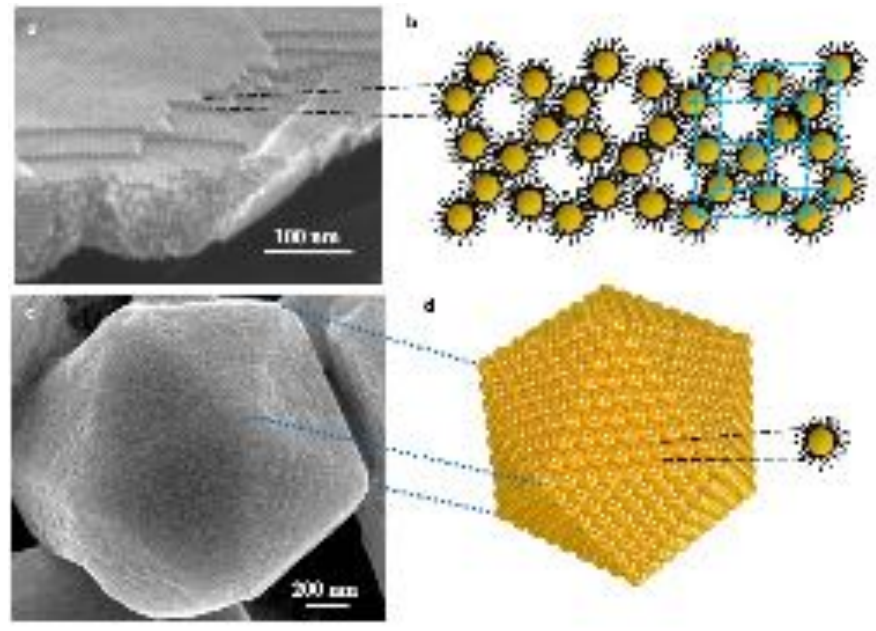

Figure. 1. Supracrystals grown under heterogeneous $(a, b)$ and homogeneous $(c, d)$ processes. SEM images of nanocrystals self-assembled layer by layer (a) and shaped supracrystals (b) of $5 \mathrm{~nm}$ Au nanocrystals coated with dodecanthiol. Scheme of such fcc supracrystals (b and d). 
Under zero solvent vapor pressure, the colloidal solution of nanocrystals is poured into a beaker with a silicon wafer at the bottom and deposited in a glove box with a volume larger than that of the beaker. With a good solvent for the alkyl chains, at the end of the solvent evaporation, a liquid-gas transition ${ }^{30}$ takes place with appearance of a lower phase highly concentrated in nanocrystals and an upper phase very diluted. At this stage, nanocrystals are self-assembled in solution. At the end of the solvent evaporation, films of nanocrystals (Fig. 2) cover most of the substrate surface previously placed at the bottom of the container. The film thicknesses are controlled by the initial nanocrystal concentration used before evaporation. The average thickness evolves from a few dozen nanometers to a few micrometers. The film surface reveals some terraces with steps having various heights from one nanocrystal thickness to several ones. The SAXS patterns show fcc crystalline ordering of nanocrystals. ${ }^{31}$
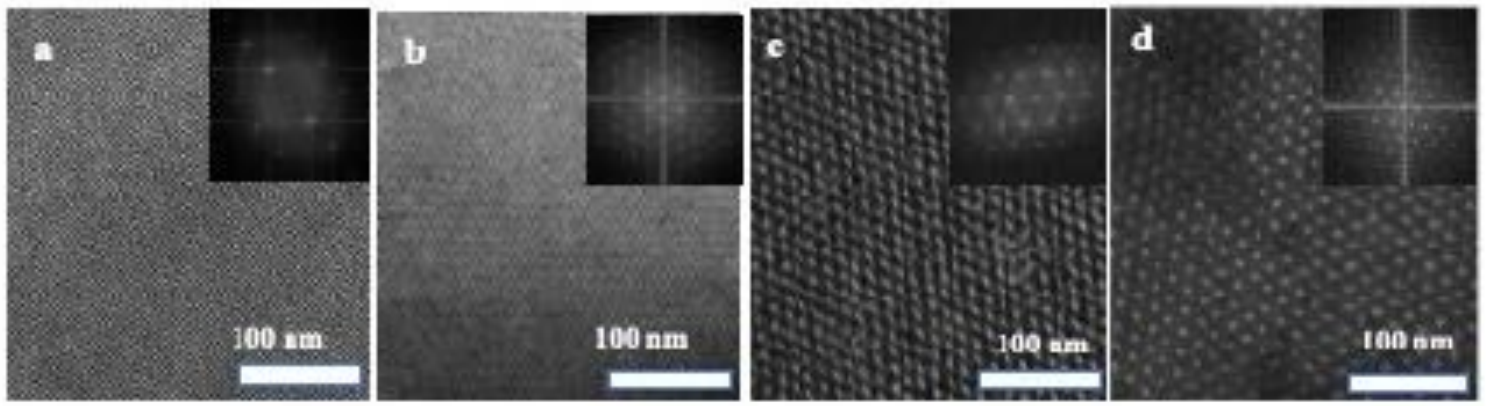

Figure 2. TEM images of thin fcc supracrystals of $\mathrm{Ag}$ nanocrystals coated with oleyamine $\left(\mathrm{C}_{18}-\mathrm{NH}_{2}\right)$ and differing by their average diameters: (a,) $2.2 \mathrm{~nm}$; (b) $4.1 \mathrm{~nm}$; (c) $8.7 \mathrm{~nm}$; (d) $12.9 \mathrm{~nm}$. Inset Fourier transform of the TEM image.

At the end of the evaporation, the drying process induces film shrinking with restriction of the film adhesion on the surface and consequently cracks formation. Figure $\mathbf{3}$ shows cracks patterns observed at various film thicknesses. The geometries of different crack patterns are observed in a very large variety of materials, over a wide range of scales with specific scaling laws. ${ }^{32-38}$ In a previous study, we demonstrated that cracks of ferrite nanocrystals films follow a scaling law over three orders of magnitude indicating a universality of crack patterns independently of their dimensions. ${ }^{39,40}$ These Cracks are observed by optical microscopy in transmission modes (Fig. 4). They form a network of channels separating islands (of a few tens of $\mu \mathrm{m}$ ) of thick film. Light coming out of channels is represented in blue while light from islands in reddish (no transmission is observed). In previously work with ferrite nanocrystals the bottom of the cracks did not show any waiting layers of nanocrystals. ${ }^{39}$ At the opposite, here by transmission microscopy (Fig. 4), we observe various color contrasts in the channels. This indicates presence of nanocrystal layers at the bottom of the cracks. This could be due to change in nanocrystal size, materials and coating agents. Consequently, the blue and white colors in the crack channels demonstrate the presence of wetting layers at the bottom of each crack. Similar patterns are observed in bright field reflection mode, cracks appearing dark due to light diffraction at the film edges (not shown here). 
The loss of uniformity in the transmission mode clearly indicates presence of several wetting layers at the bottoms of the cracks.
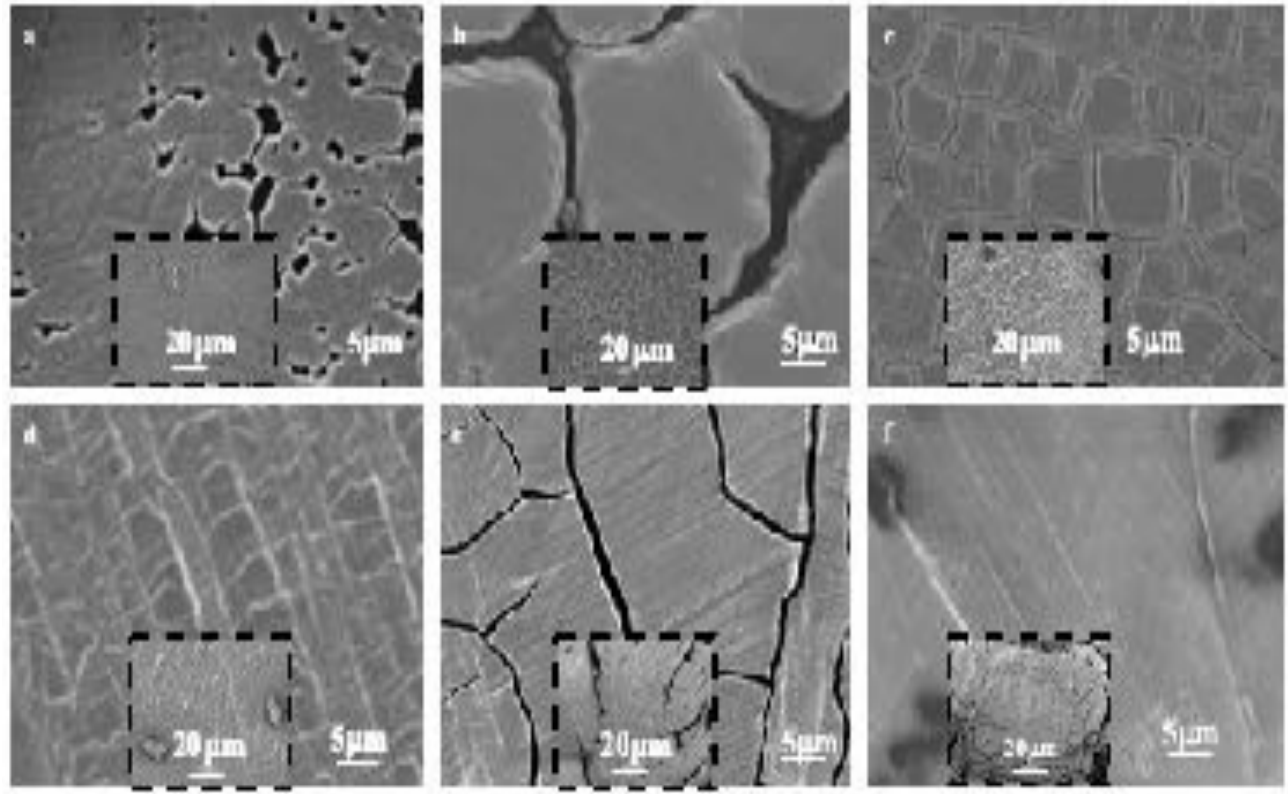

Figure 3. SEM images of supracrystal films of $8.2 \mathrm{~nm} \mathrm{Ag} \mathrm{nanocrystals} \mathrm{with} \mathrm{various} \mathrm{thicknesses.} \mathrm{(a)} \mathrm{0.2-0.6} \mu \mathrm{m}$, (b) 0.4-1.0 $\mu \mathrm{m}$, (c) 0.6-1.4 $\mu \mathrm{m}$, (d) $0.9-1.6 \mu \mathrm{m}$. (e) $1-1.4 \mu \mathrm{m}$ (f) $1.3-1.9 \mu \mathrm{m}$.

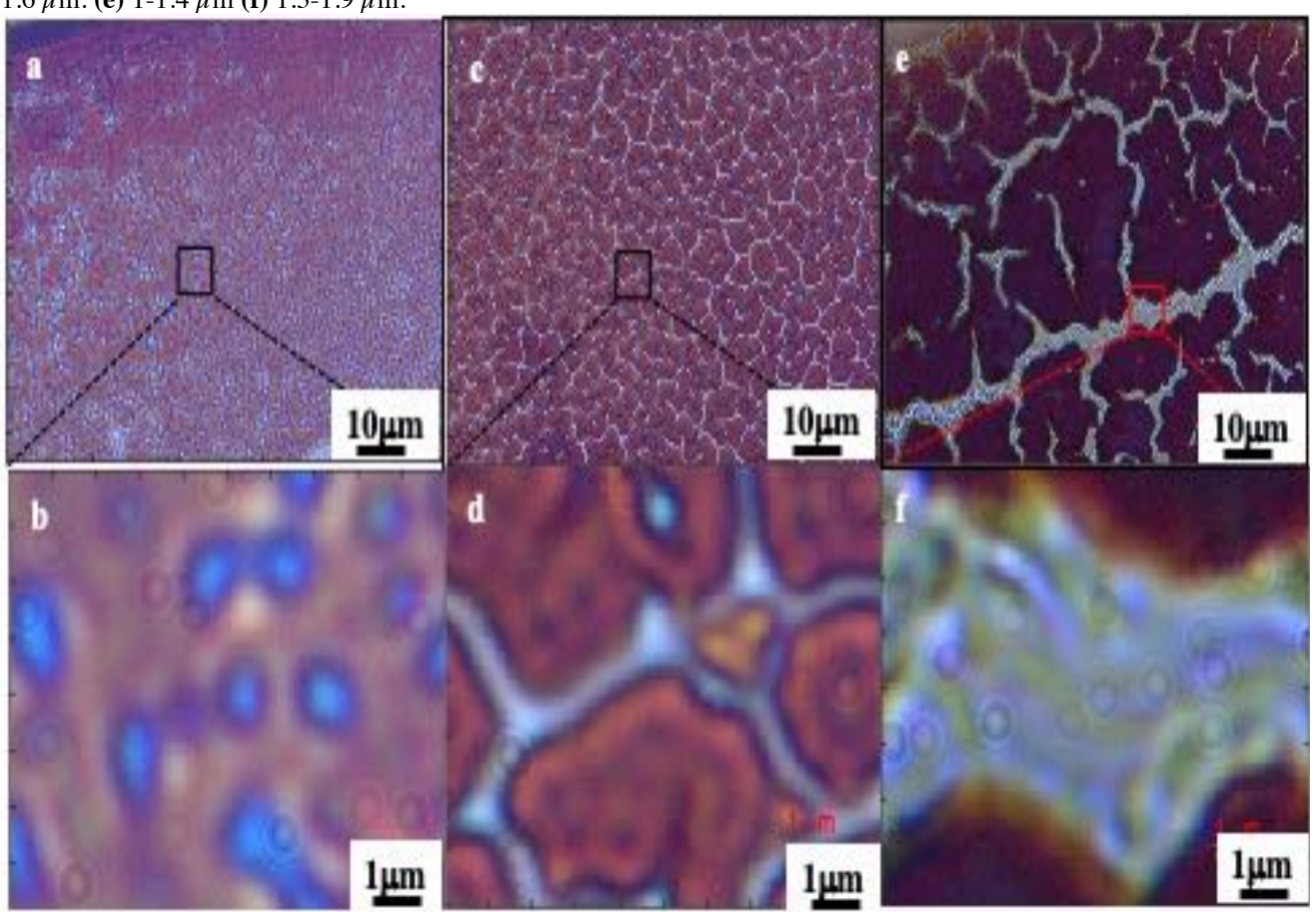

Figure 4. Optical micrographs in transmission mode of the 0.2-0.6 $\mu \mathrm{m}$ supracrystal film. (a, b,), 0.4-1.0 $\mu \mathrm{m}(\mathbf{c}, \mathbf{d})$ and 1.0-1.4 $\mu \mathrm{m}(\mathbf{e}, \mathbf{f})$.

To produce water dispersive “clustered" structures ${ }^{\mathbf{2 8 , 2 9}}$ from hydrophobic Au nanocrystals, we need to build up an "organic molecular parachute". For this purpose, we use two concepts:

i) Bad solvents for the coating agents: $\mathrm{Au}$ shaped supracrystals of $5 \mathrm{~nm} \mathrm{Au}$ nanocrystals coated with octadecanethiol are produced by controlling the colloidal crystal growth process: Addition of traces of a nonsolvent such as ethanol to an Au colloidal solution subjected to solvent saturation induces a marked increase in 
the attractive interactions between nanocrystals and consequently a homogeneous growth process. ${ }^{17}$ The SEM images of the collected Au precipitates (Fig. 5a) show formation of well-defined aggregates with various shapes and a rather large size distribution. Most of these shaped aggregates are characterized by a flat surface with welldefined facets in hexagonal symmetry, while others present some defects but no cracks are observed. The SAXS experiments show formation of fcc Au supracrystals.

ii) Interdigitation processes and production of multi-walled structures: For this purpose, we use a concept, developed several years ago, to produce thermodynamically stable assemblies of surfactant molecules to build up supra-aggregates: '1 The best interdigitation process is obtained with long tail surfactant molecules and large head group surface area. 42 Because voids are energetically unfavorable, the dipalmitoylphosphatidylcholine (DPPC) alkyl chains and the $\mathrm{C}_{18}$ chains of PEG-2000-DOPE fill them up. This induces strong interactions between DPPC, PEG-2000-DOPE and nanocrystals coated with $\mathrm{C}_{18} \mathrm{H}_{37} \mathrm{SH}$. The PEG2000-DOPE, hydrophilic chain $\left(\mathrm{C}_{91}\right)$ makes bridges from one vesicle to another and consequently plays the role of "parachutes" preventing gravity processes.

The SEM (Fig. 5b) and the corresponding TEM (Fig. 4c) images reveal the structure of individual composites, with presence of large vesicles attached to the supracrystal surface and drape-like structures linked to the supracrystal.

Hence, heavy shaped Au supracrystals of 5nm nanocrystals are associated via interdigitations between the alkyl chain $\left(\mathrm{C}_{18} \mathrm{H}_{37}-\mathrm{SH}\right)$ used to coat nanocrystals, the biological surfactant such as DPPC and PEG-2000-DOPE characterized by both long alkyl chains and polar chain favouring hydrogen bounding. This is represented by the cartoon shown on Figure 6. 

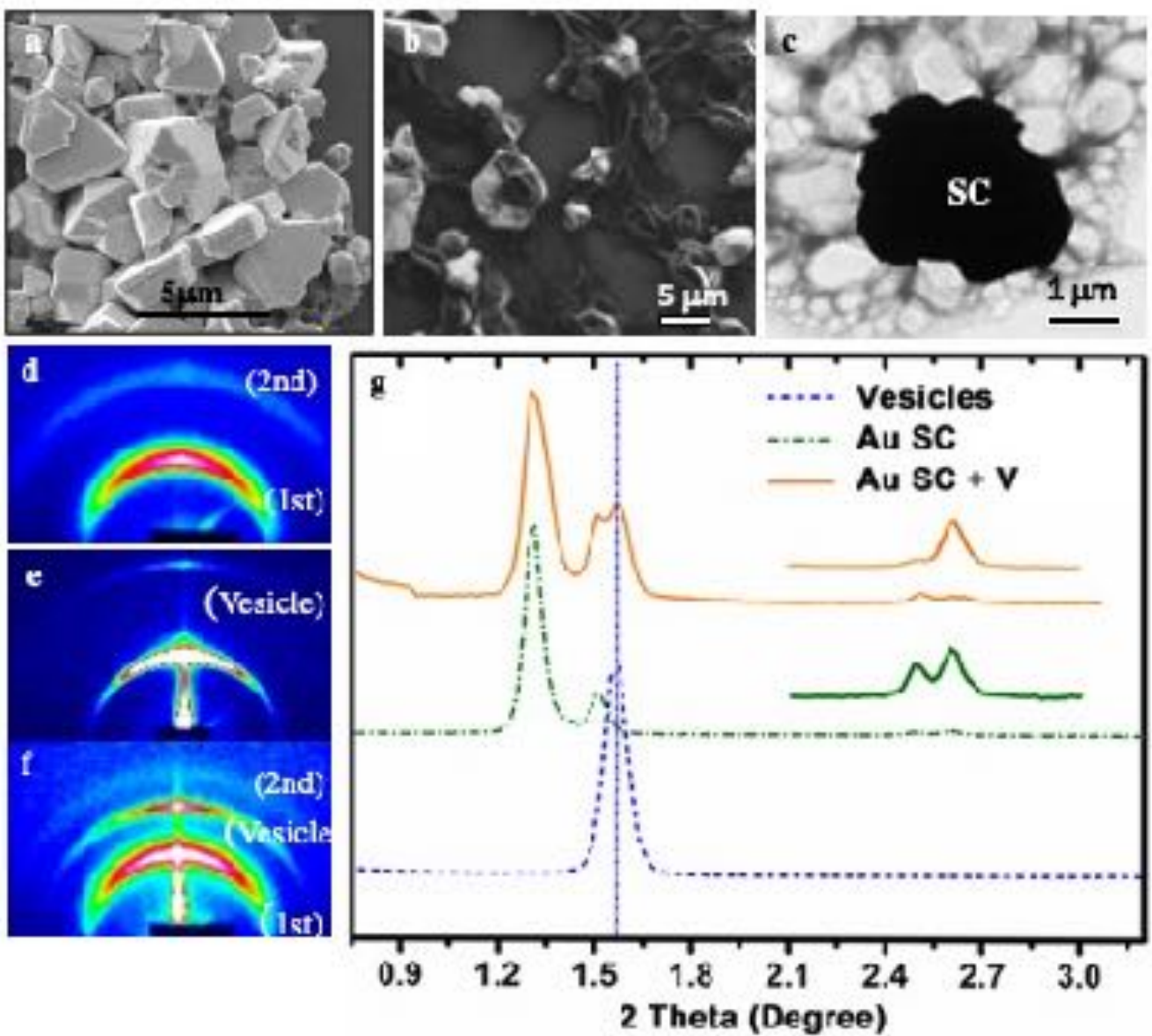

Figure 5 (a) and (b) are the SEM images of Au shaped supracrystals before (a) and after (b) DPPC/PEG-DOPE treatments. (c) TEM image corresponding to image of (b) showing that the vesicles are closely attached to the supracrystals forming cap-like structures. (d) SAXS characterization of the supracrystals, (e) the vesicles and (f) the clustered structure (g) profile the different SAXS patterns: The two red peaks in (f) can be attributed to the diffraction of the supracrystals and vesicles, respectively.

The patterns of structural studies, performed by SAXS, are acquired on shaped supracrystals before (Fig. 5d) and after (Fig. 5f) DPPC/PEG-DOPE treatment. The supracrystal pattern (Fig. 5d) is attributed to the diffraction of related planes of supracrystals with the edge-to-edge interparticle distance of $2.8 \mathrm{~nm}$. The pattern corresponding to the supracrystal-vesicle composite shows well-defined rings (Fig. 5f). The new ring appearing in the diffraction pattern is attributed to the presence of vesicles. To support such statement, a blank experiment is performed, in absence of supracrystals, with DPPC/PEG-DOPE molecules. After hydration, the solution is dried and the SAXS pattern (Fig. 5e) shows a strong signal due to the diffraction of periodic vesicle bilayers. The calculated bilayer distance is $5.7 \mathrm{~nm}$, which agrees with the thickness of the liposome shell. ${ }^{43}$ The profile (Fig. 5g) clearly shows that the diffraction peaks of the composite are a combination of the peaks of isolated $\mathrm{Au}$ supracrystals and of vesicles indicating that the DPPC/PEG2000-PE vesicles strongly interact with Au supracrystals. Similar data are obtained with Co instead of Au nanocrystals. 


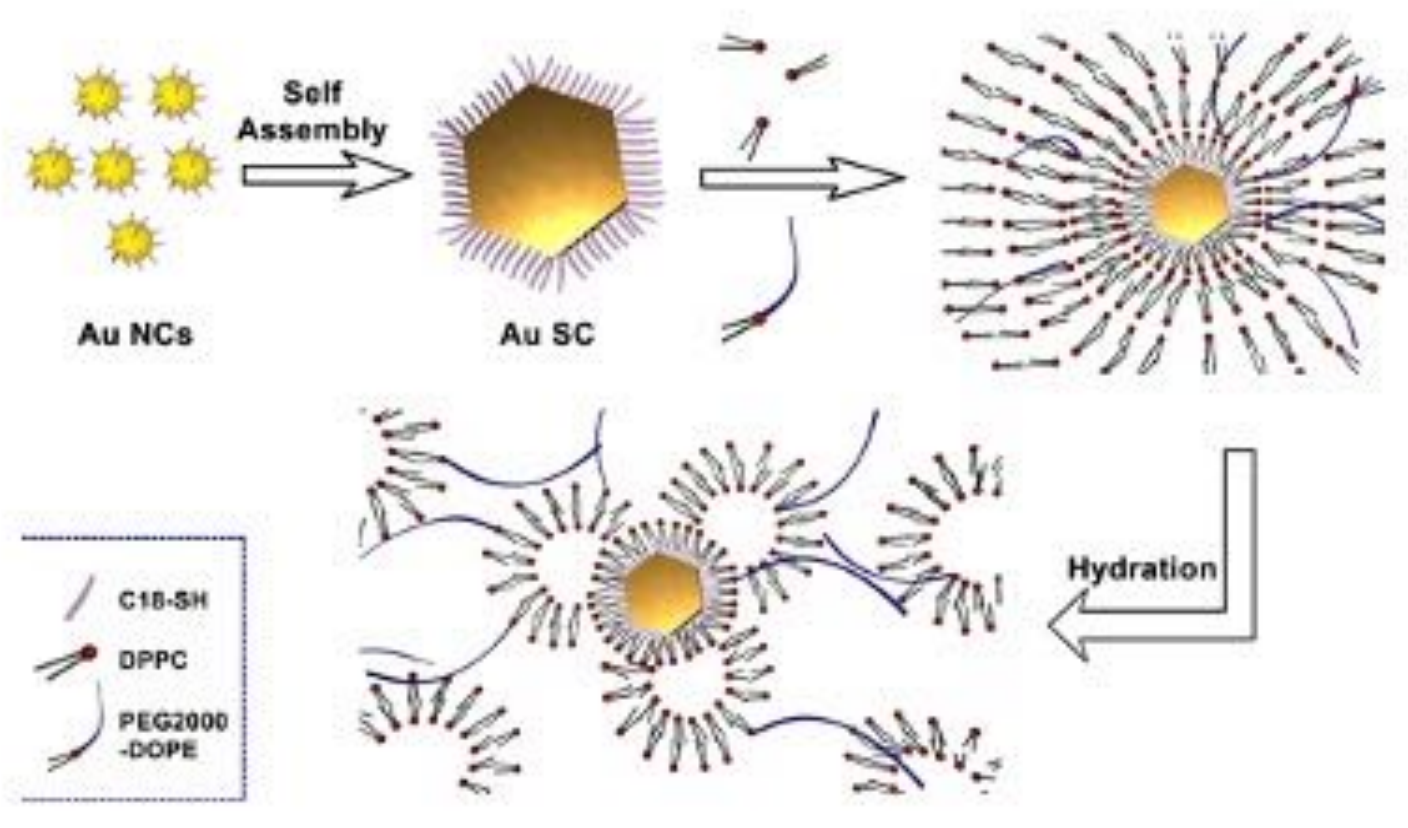

Figure 6. Cartoon of the procedure used to growth shaped supracrystals of hydophobic coating agent treated with organic molecules such as DPPC and PEG-2000-DOPE. This permits to disperse shaped hydrophobic supracrystals in aquoeus solution.

\section{Optical properties of Ag supracrystals 31,44}

Let consider dried thin fcc supracrystalline films (less than 7 layers) of Ag nanocrystals coated with oleic acid differing by their sizes and deposited on a substrate. Their optical properties are compared to nanocrystal dispersed in toluene.

On increasing nanocrystal size, the Localized Surface Plasmon Resonance (LSPR) peaks are shifted toward high energy when nanocrystals are dispersed in solution whereas to lower energy when they are self-assembled in 3D superlattices (Fig. 7). This is attributed to dipolar interactions between nanocrystals. 11,23,45

On increasing the number of nanocrystals layers, the LSPR spectra should reach saturation, which explains why most of the optical properties of supracrystals were investigated for less than ten layers. $23,26,45-50$ One attempt, in limit of Beer Lambert law, was performed on supracrystals having with 30 to 35 layers. ${ }^{50}$ By using Effective Medium Theory (EMT) without features on their surface and by taking into account the crystalline structure of the film (fcc) with $8.2 \mathrm{~nm}, 2.8 \mathrm{~nm}$ and 0.3 as average nanocrystal diameter, nanocrystal interparticle distance and filling factor respectively, the calculated LSPR spectra of homogeneous supracrystal film (flat surface) differing by their thicknesses, from 28 to 180 nanocrystal layers, reach saturation in the wavelength range from $300 \mathrm{~nm}$ to $550 \mathrm{~nm}$. At larger wavelength, the transmission intensity through the supracrystal films decreases with increasing the film thickness and a wider linewidth is observed. Figure $\mathbf{8}$ shows transmission spectra for various film thicknesses. For fcc supracrystal films formed by 27 to 78 layers, a direct measurement of absorption, reflection, and transmission is still possible (Fig. 8a). By increasing the number of layers from 27 to 180, the transmission spectra of Ag supracrystal film (Figs. 8 a-d, dashed line) reveal a decrease in the transmission with an increase in 
the notch full-width at half maximum (FWHM) as the film thickness increases. Hence the calculated spectra totally disagree with the experimental data.

According to the optical micrographs in transmission mode shown on Figure 4, we assumed presence a wetting layer of nanocrystals with a constant thickness at the bottom of each crack. Unfortunately, the experimental and the calculated spectra do not agree. This could be attributed to the uniform thickness assumed in the calculation. This is supported by the fact that Figure 4 shows a rather inhomogeneity in the transmission inside the channels. In the calculations presence of steps having various thicknesses by assuming at the bottom of each crack is introduced. In the total transmission calculation, the thickness and the weighted sum of the transmissions of each zone are the two free parameters taken into account. Figure 8 reveals the excellent agreement between calculations accounting for the cracks and experiments for films of various thicknesses $(0.2-0.6 \mu \mathrm{m}, 0.4-1 \mu \mathrm{m}$, 0.6-1.4 $\mu \mathrm{m}$, and 0.9-1.6 $\mu \mathrm{m})$. The solid red, green, and cyan lines represent the transmission spectra of different wetting layers that are introduced in the cracks for each film. The blue trace is the sum of all coloured lines.

From these data, we conclude that cracks dominate the optical response of the films of different thicknesses. Wetting layers with different thicknesses, sitting at the bottom of the cracks explain the optical properties observed with thick fcc films of Ag nanocrystals with a large number of layers. From these data it seems impossible, with dried fcc supracrystals, to observe any collective optical modes

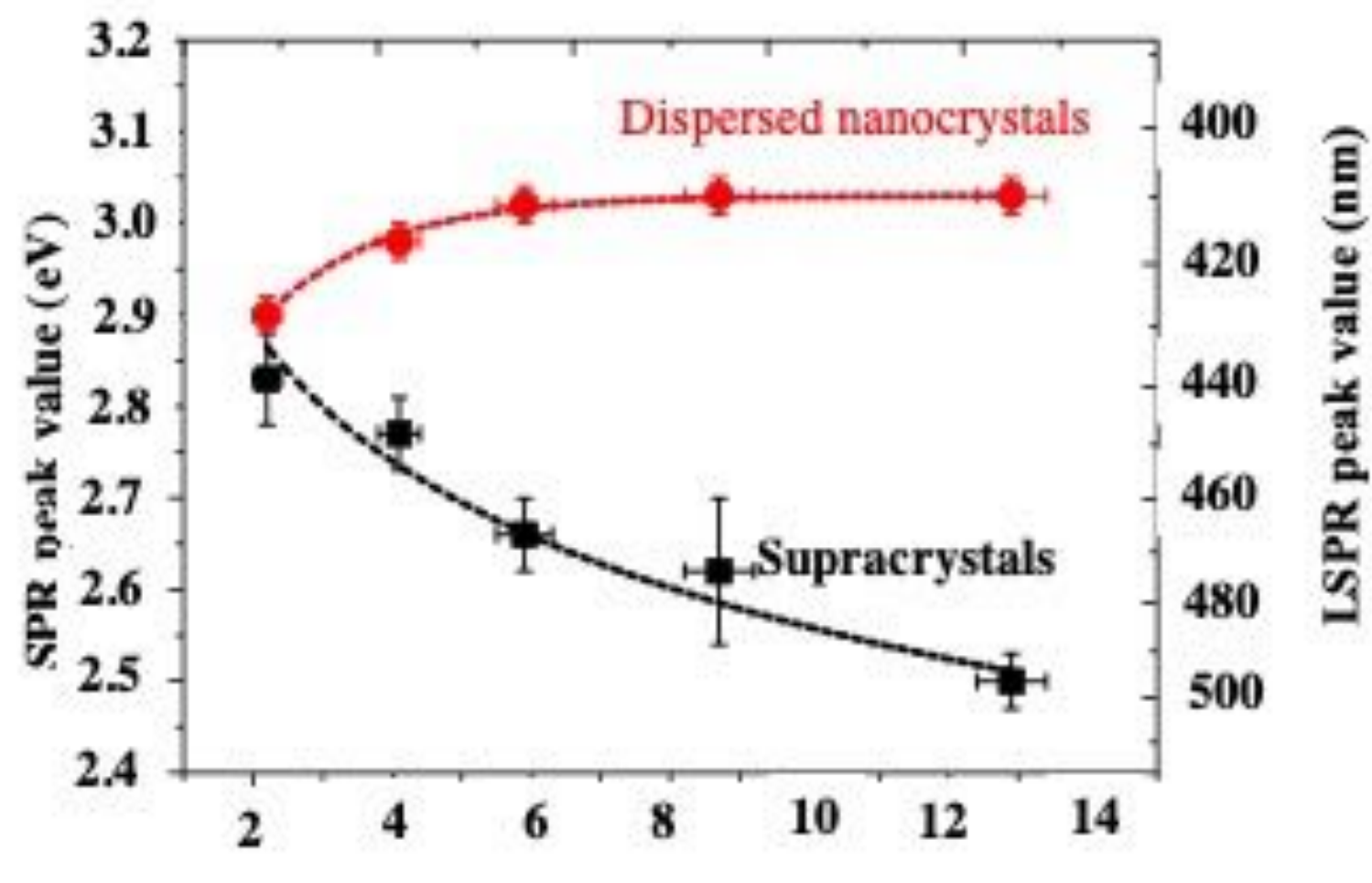

\section{NCs diameter (nm)}

Figure 7. LSPR peak position values of dispersed nanocrystals (red dots) and corresponding supracrystalline films (black squares) as a 

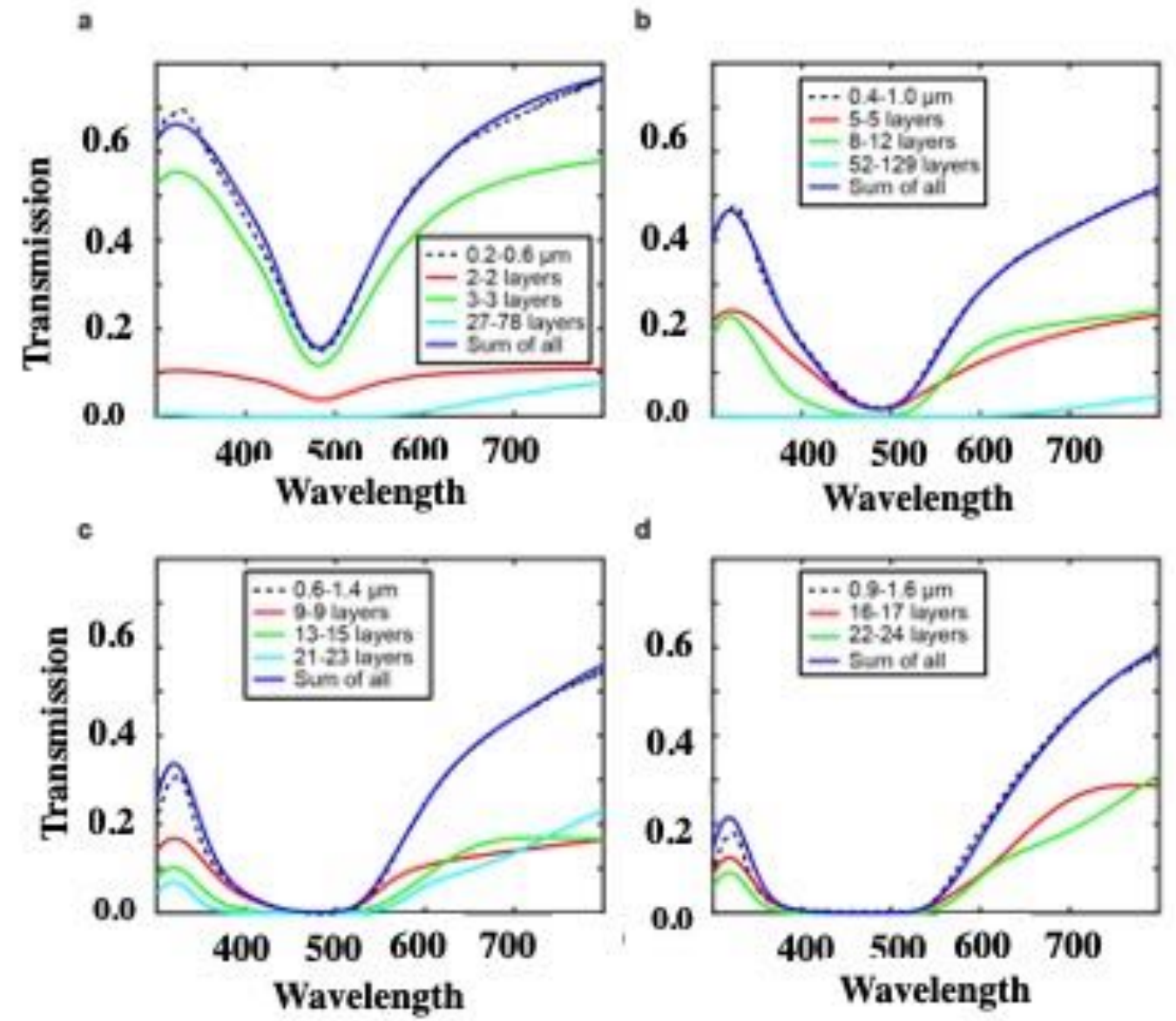

Figure 8 Calculated (blue solid traces) and experimental (dashed traces) transmission spectra of 8.2-nm Ag NCs supracrystal films with various thicknesses. (a) $0.2-0.6 \mu \mathrm{m}$, (b) $0.4-1 \mu \mathrm{m}$, (c) $0.6-1.4 \mu \mathrm{m}$, and (d) $0.9-1.6 \mu \mathrm{m}$. The calculated spectra account for wetting layers with different thicknesses. Colors correspond to different number of monolayers.

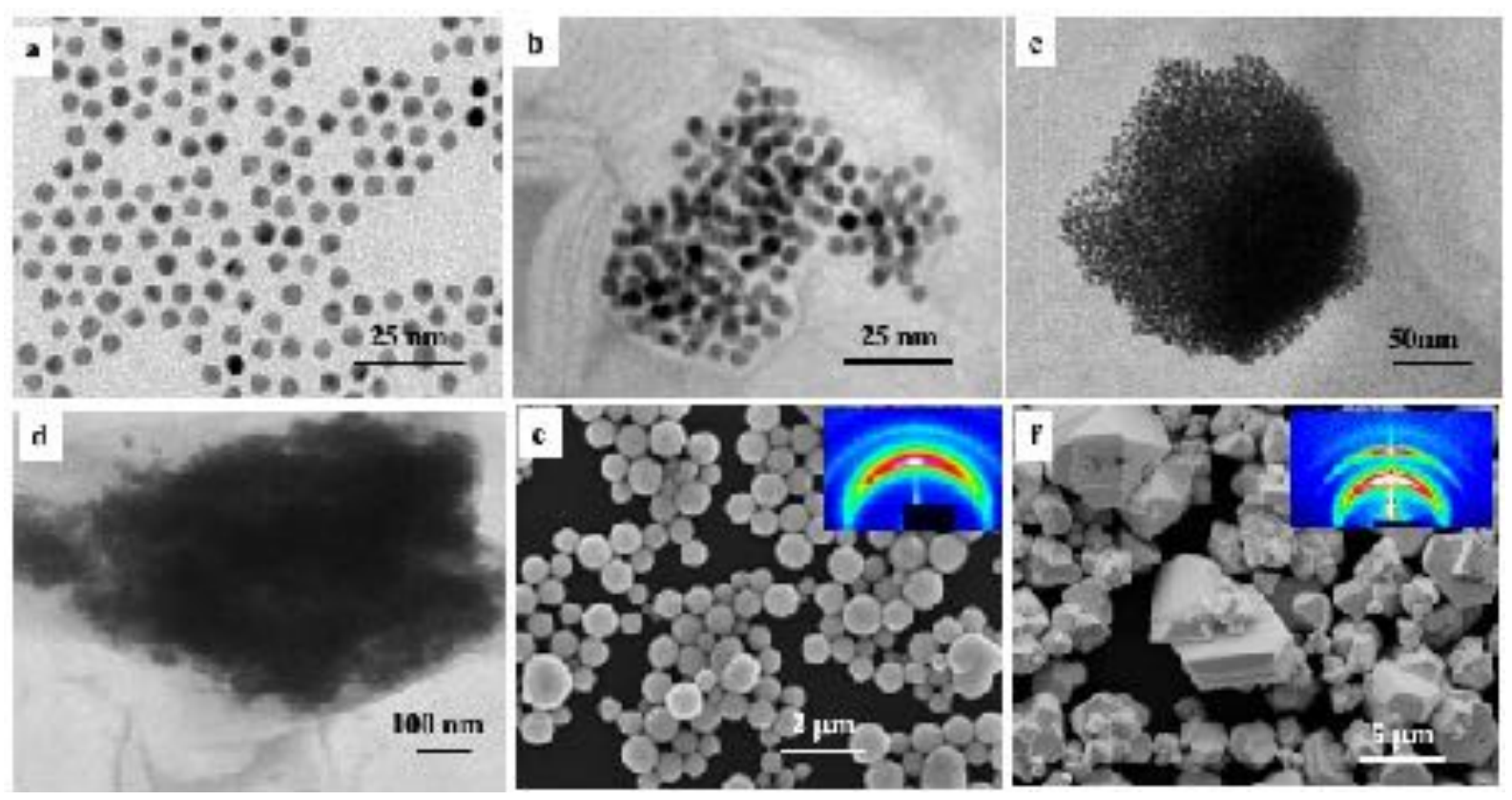


Figure 9. TEM (a, b, c, d) and SEM (e, f) images of 5.5nm Au supracrystals (a) either isolated (a) closed packed $(b, c, d)$ or self assembled $(e, f)$ in fcc crystalline structures. The sizes are $52 \mathrm{~nm}$ (b), $125 \mathrm{~nm}$ (c), $134 \mathrm{~nm}$ (d), $413 \mathrm{~nm}(\mathrm{e})$ and $611 \mathrm{~nm}(\mathrm{f})$.

Instead of studying the optical properties of dried fcc supracrystals of $5 \mathrm{~nm} \mathrm{Ag} \mathrm{nanocrystals} \mathrm{deposited} \mathrm{on} \mathrm{a}$ substrate, let consider fsupracrystals dispersed in aqueous solution. Here we strudy supracrystals of $5.5 \mathrm{~nm} \mathrm{Au}$ nanocrystals. ${ }^{29}$ On controlling the nucleation and growth times, it is possible to produce either aggregates or supracrystals differing by their sizes from $52 \mathrm{~nm}$ to $600 \mathrm{~nm}$. These aggregates and/or supracrystals, dispersed in aqueous solution, are characterized by a rather large size distribution when that of the building blocks is low $(<6 \%)$. Note that for small aggregates (Figs. 9b, $\mathbf{c}$ and d), the Au nanocrystal assemblies are not well ordered. At the opposite, the largest aggregates (Fig. 9e and f) are fcc supracrystals (413nm and $611 \mathrm{~nm})$. The assembly size distribution is obtained from TEM or SEM images of almost 100 aggregates. The absorption spectra of the assemblies of $5 \mathrm{~nm}$ Au nanocrystals differing by their sizes, shown on Figure 9, are red shifted on increasing their diameters with a shoulder around 530nm (Fig. 10).
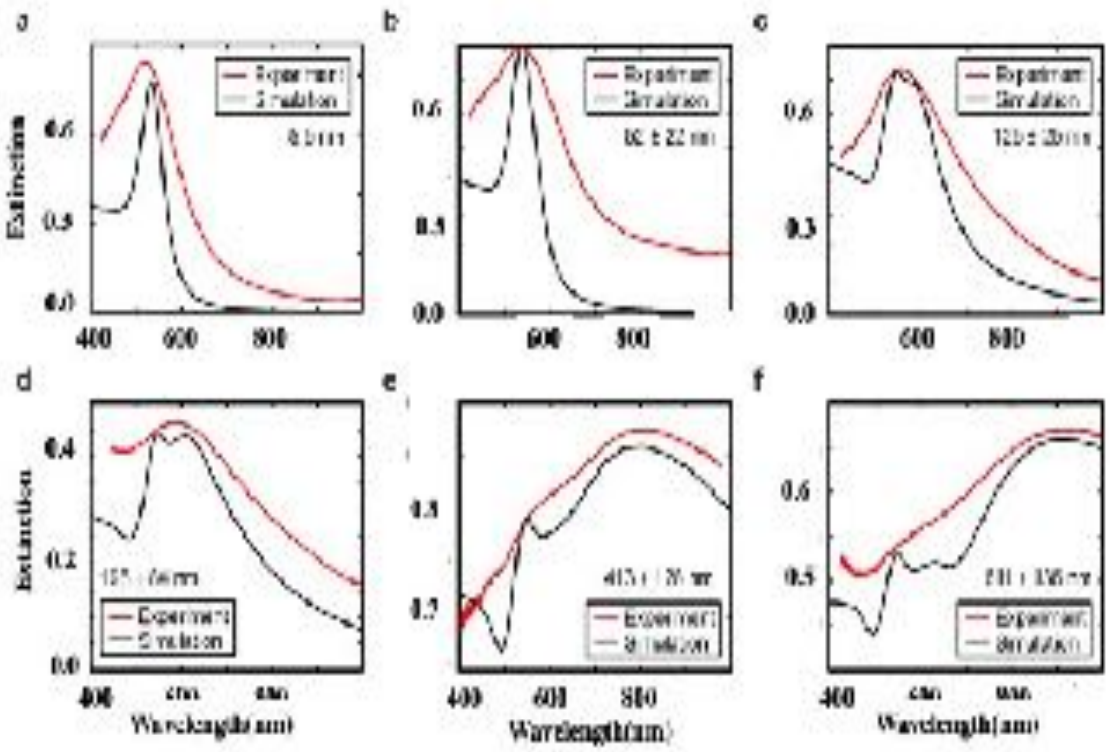

Figure 10. Extinction spectra for isolated Au nanocrystals and assemblies with various sizes. (a) $5.5 \pm 0.4 \mathrm{~nm}$ Au nanocrystals; Au aggregates with sizes of (b) $52 \pm 22 \mathrm{~nm}$, (c) $125 \pm 25 \mathrm{~nm}$, (d) $198 \pm 84 \mathrm{~nm}$; Au supracrystals with sizes of (e) $413 \pm 128 \mathrm{~nm}$ and (f) $611 \pm 138 \mathrm{~nm}$. The average sizes were used to fit the measured data.

To explain such large difference in the absorption spectra with aggregate size, we need simulations. To do it, we first calculate the effective dielectric function of $\mathrm{Au}$ assemblies by using Maxwell-Garnett effective medium theory (EMT). ${ }^{51}$ Then Mie theory is used to calculate the spectral response of Au assemblies dispersed in water. The distribution over the size of assemblies is deduced from SEM images and accounted for in our calculations.

Figure 10 shows that the experimental spectra, recorded at various aggregate sizes, agree reasonably with the calculated ones. Hence, the calculated absorption spectra are decomposed in two well-defined spectra: At high energy, the absorption spectrum is well resolved with a rather small bandwidth whereas the spectrum at low 
energy is very broad. This is observed whatever the aggregate size. However, on increasing the aggregate size, the high energy peak remains constant with a maximum spectrum centred at $530 \mathrm{~nm}$. Conversely, the spectrum at low energy is red-shifted on increasing the aggregate size. Even though the size distribution is rather large, the maximum of the calculated broad peak linearly depends of the aggregate size (Figure 11). From the unchanged position of the high energy peak in the assemblies compared to dispersed Au nanocrystals in aqueous solution (Figs. 10a and 11a), we conclude that the peak centred at $530 \mathrm{~nm}$ is due to Localized Surface Plasmon Resonance (LSPR) of $5 \mathrm{~nm}$ Au nanocrystals. This clearly indicates that the LSPR of isolated nanocrystals is retained when they are self-assembled either in small aggregates or in fcc supracrystals. Hence the fingerprint of nanocrystals in supracrystals is retained. The linear dependence of the second and broad second peak with the aggregate size indicates that the shift toward low energy is due to the aggregate size. Consequently, it can be attributed to collective modes due to the assemblies. However, we note that with small assembly size, Au nanocrystals are closed together and not assembled in crystalline structures whereas the larger one $(413 \mathrm{~nm}$ and $611 \mathrm{~nm}$ ) are fcc supracrystals. From Figure 11 no disruption is observed between data obtained with slightly disordered aggregates and the supracrystals. This permits to assume such change in the peak position at low energy is due to dipolar interactions between nanocrystals. Hence, here we claim that nanocrystals closed together induces collective modes when they are dispersed on aqueous solution

To conclude Au nanocrystals assembled in aggregates or in supracrystals dispersed in water show a fingerprint of isolated nanocrystals and collective modes whereas with Ag nanocrystals self -assembled in fcc supracrystals and deposited on a substrate (dried system) such behaviours cannot be observed. ${ }^{44}$ 


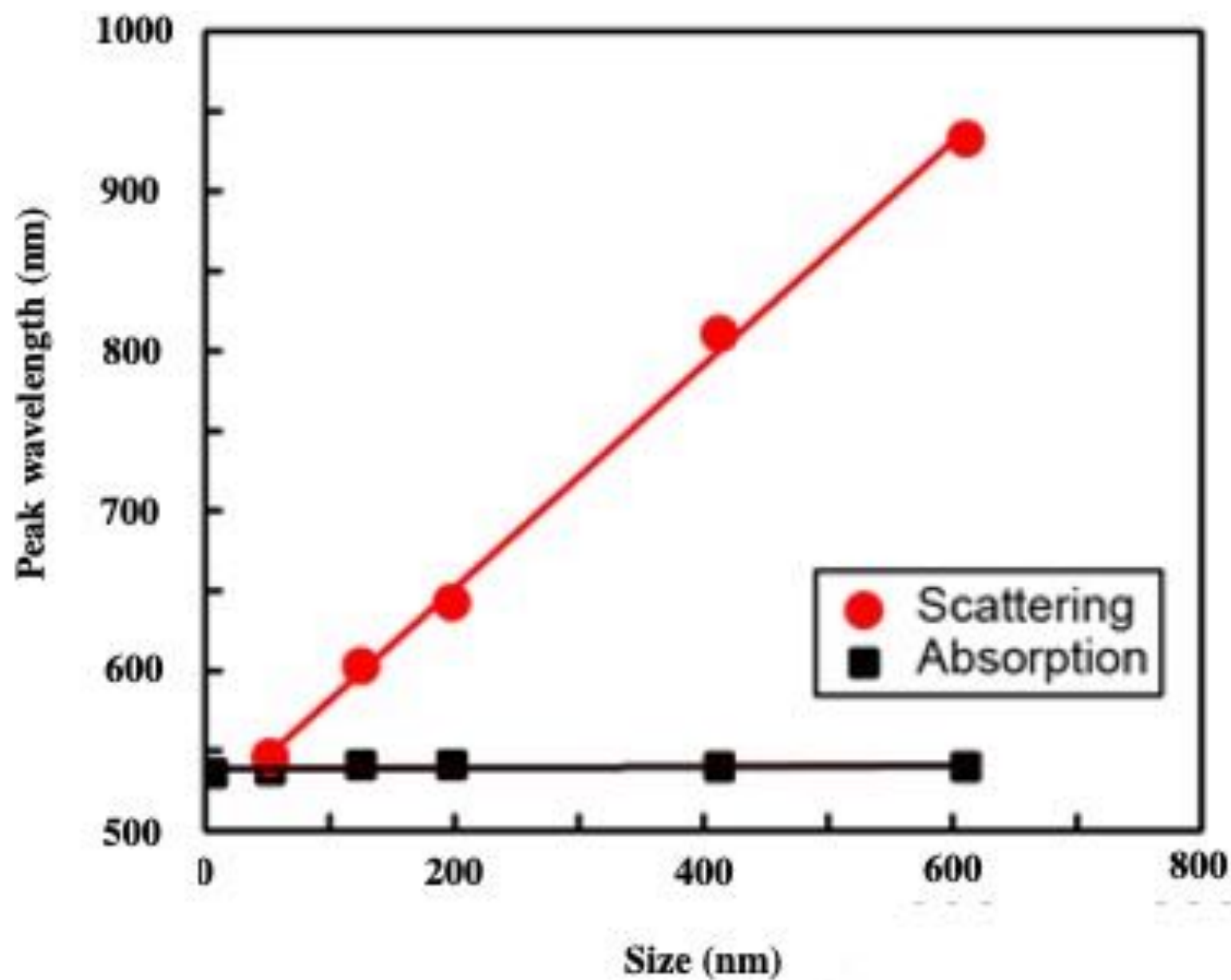

Figure 11. Variation of the peak wavelength as a function of the assembly size: The black curve illustrates the absorption peak of Au that is present for all assemblies. The scattering peak represented in the red curve red shifts as the assembly size increases.

\section{Breathing mode process.}

In dried systems, as already mentioned, the substrate temperature controls the ordering of nanocrystals. Hence, by increasing the substrate temperature from $10{ }^{\circ} \mathrm{C}$ to $25^{\circ} \mathrm{C}$, the $\mathrm{Ag}$ nanocrystals ordering evolves from disordered film considered as amorphous to small fcc supracrystals and finally to a long-range Ag fcc supracrystals. The Stokes-anti Stokes Raman spectra, characterized by the quadrupolar modes, of amorphous film behaves as the Raman spectrum of a collection of nanocrystals, $\mathbf{5 2}$ indicating a good agreement between amorphous film of Ag nanocrystals deposited on a substrate and a collection of the same nanocrystals dispersed in solution. With both "large and small" fcc supracrystals a shifted to low frequencies compared to amorphous film is observed and is attributed to the Lorentz field effect. The major difference between the two types of supracrystals (small and large ones) concerns the width of the quadrupolar lines. With "large" supracrystals, they are perfectly superimposed to those of amorphous film whereas with "small" supracrystals (size $\lambda \leq \lambda / 10$ where $\lambda$ is the wavelength of incident line) the quadrupolar lines are narrower than those of amorphous films. Such changes in the Raman scattering spectra were attributed to nanocrystals vibrating coherently in a supracrystal. ${ }^{24}$ In conclusion, nanocrystals do not move and vibrate at fixed positions. This claim was supported by mathematical calculation from which the "small" supracrystal Raman intensity is proportional to the square of 
intensity of its corresponding amorphous film. These data clearly indicated inter-nanocrystal coherence inside fcc supracrystals.

Let consider the water dispersed Au "clustered" structure with average size of $196 \pm 86 \mathrm{~nm}$ (around 14 layers) characterized by a large peak centred around 700nm (Fig. 10b). The energy flow upon light irradiation is investigated by pump-probe experiments. At long-times scales the differential transmission $(\Delta T / T)$ spectra, investigated by pump-probe experiments and recorded as a function of time and of the probe wavelength $(\lambda)$, a mechanical oscillation period is observed (Fig. 12). This period, attributed to the breathing mode of the whole assembly, is around of 300ps. ${ }^{\mathbf{5 3}}$ The calculated period is given by $T=2 D / \mathrm{v}_{s}$ where $D$ is the average diameter (198 $\mathrm{nm} \pm 84 \mathrm{~nm}$ ) of the assembly and $\mathrm{v}_{\mathrm{s}}$ the speed of sound inside the supracrystal. By using the same technique, we observed coherent longitudinal acoustic phonons in small Co supracrystals of $7.1 \mathrm{~nm}$ Co nanocrystals used as building blocks. ${ }^{54}$ These experiments allowed to estimate, at room temperature, the speed of sound in the supracrystal to $1235 \pm 12 \mathrm{~m} . \mathrm{s}^{-1}$. If we assume the speed of sound remains similar with Au than Co supracrystals, the resulting oscillation period of the Au "clustered" structure is $236 \pm 77$ ps consistent with experimental data. These results provide a clear indication from which the coherent vibration of nanocrystals in a supracrystal can be directly observed by using water soluble hybrids whereas an indirect process is needed for dried supracrystals.

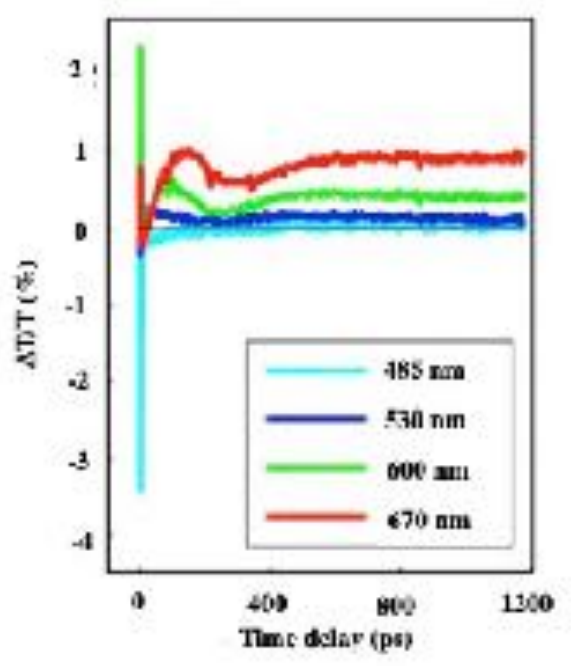

Figure 12 Dynamics of the $\Delta T / T$ at selected probe wavelengths, on nanometer time scales of energy flow in the Au clustered structures.

\section{Nanoheater.}

Let consider dried either Au supracrystals deposited on a substrate. Time-resolved differential transmission $(\Delta \mathrm{T} /$

T) obtained by using fentosecond pump-probe technique shows the breathing mode $(l=0)$ of nanocrystals. The decay reaches zero around 10ps (Fig. 13). No other transient is observed.53 


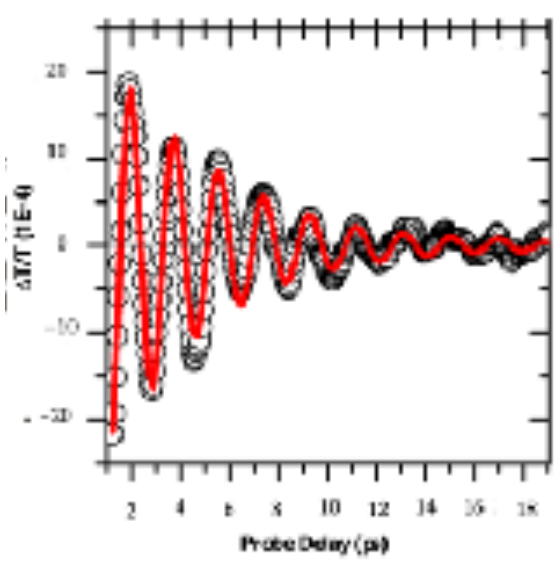

Figure 13. DT/T time traces extracted from $2 \mathrm{D}$ maps at selected wavelength $1_{\text {probe }}=560 \mathrm{~nm}$ for $10.6 \mathrm{nnm}$ Au nanocrystals

By using the same Au "clustered" structures as described above (196nm, Fig. 10d). at the initial time scale of ten picoseconds, the typical $\Delta T / T$ map is due to the transient spectra of isolated NCs at various probe wavelengths. They are dominated by shift and broadening of the plasmonic resonances as observed previously. ${ }^{54}$ These spectra exhibit a decay constant of few picoseconds (Fig. 14a), corresponding to the timescale of electron-phonon scattering in noble metals ${ }^{56}$ indicating that the fingerprint of dispersed nanocrystals used as building blocks is kept. Similar decay was obtained with supracrystals deposited on a substrate (dried system), Fig. 13
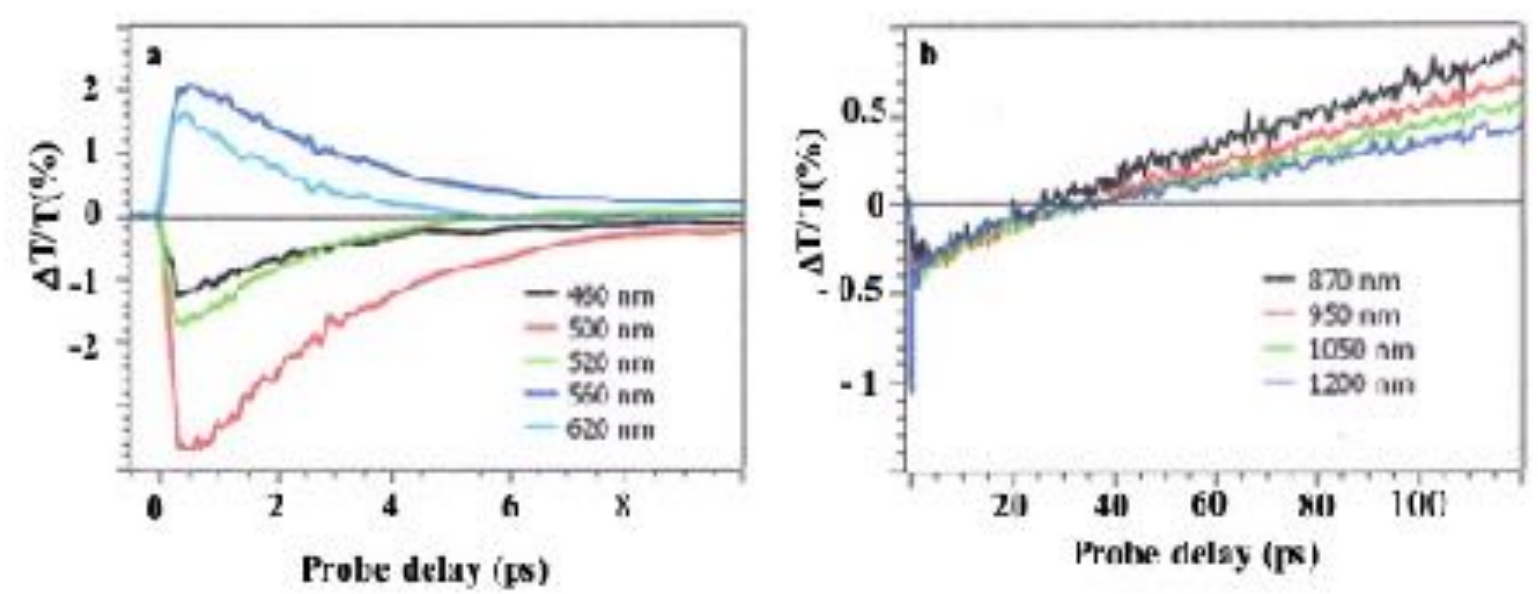

Figure 14. Dynamics of the $\Delta T / T$ at selected probe wavelengths, at the two different time scales of energy flow in the Au clustered structures $(a, b)$. From these data water dispersed Au "clustered" structure can be operated as very efficient nanoheaters whereas it was not observed in dried Au supracrystals.

At longer times scales, the $\Delta T / T$ map of Au "clustered" structures (Fig. 14b) exhibit very distinct features which, to the best of our knowledge, have not been reported for any nanostructures: instead of a monotonic decrease over time, we observed the build-up of a positive signal which is red shifted by about 100nm compared to early spectra. At the nanosecond time scale, after the build-up attributed to the fingerprint of mechanical oscillation, the signal remains constant Figure 12. To explain the origin of such unexpected transient optical response at the 100-ps timescale, then remaining constant at the nanosecond time scale, a model based on the large penetration depth of visible light in the supracrystal structure is proposed to simulate the optical experiments. ${ }^{57}$ The power absorbed per unit volume, for $400 \mathrm{~nm}$ as excitation wavelength, is more uniform than in Au nanocrystal structure 
of the same diameter. The pump absorption initiates a chain of energy transfer processes. The coating agent used to coat the nanocrystals, even though not directly absorbing, acts as an internal reservoir for efficient accumulation of energy within few hundreds of picoseconds. The very good agreement between experiments and the model confirms a collective regime of photo-temperature generation enabled by the assembling.

\section{Conclusions.}

Here, we pointed out that, the specific intrinsic properties induced by light markedly depend on the supracrystal environment. We compare the light interactions with supracrystals of hydrophobic metal nanomaterials when they are either deposited on a substrate or dispersed in aqueous solution.

Collective photonic modes are observed with supracystals dispersed in aqueous solution (Au "clustered" structures) whereas, with dried supracrystals, the optical properties are governed by cracks through the wetting layers present on the substrate. This clearly shows the influence of the environment on the intrinsic properties. Note that no cracks are observed with shaped supracrystals used to disperse them in aqueous solution.

Coherent breathing modes of nanocrystals in supracrystal are observed in both conditions. However, the period can only be evaluated with a good agreement between the measure and calculated period whereas with dried supracrystals it cannot be measured directly and is supported by calculation. Note that such breathing modes could explain the data obtained previously, at low temperature, by scanning tunneling microscopy/scanning tunneling spectroscopy (STM/STS) obtained with Au supracrystals deposited on a substrate. It has been possible to image and measure the electronic properties of the supracrystals having the thicknesses of several micrometers. Usually no tunneling effect can be obtained with such thick material. However, if we assume that when the supracrystals is subjected to current, the nanocrystals could breathe coherently favoring the pathway of electron from the tip to the substrate.

In aqueous solution, we show that the hybrid structures behave as nanoheaters. whereas it is not observed in dried supracrystals.

\section{Conflicts of interest}

No conflicts of interest

\section{Acknowledgements}

MPP thanks Prs G.Cerullo, G. DelLa Valle, J.L. Pelouard, J. Wei, N.Yang, Z.Yang,

\section{References}

1. J.V. Sanders. Color of Precious Opal Nature 1964, 204, 1151-1153.

2. L. Motte, F. Billoudet, F. and M.P. Pileni. Self-Assembled Monolayer of Nanosized Particles Differing by Their Sizes. J. Phys. Chem. $1995,99,16425-16429$

3. C.B. Murray, C.R. Kagan and M.G. Bawendi. Self-Organization of CdSe Nanocrystallites into Three-Dimensional Quantum Dot Superlattices. Science 1995, 270, 1335-1338.

4. R.L. Whetten, M.N. Shafigullin, J.T. Khoury, T.G. Schaaff, J. Vezmar, M.M. Alvarez and A. Wilkinson Crystal Structures of Molecular Gold Nanocrystal Arrays.Acc. Chem. Res. 1999, 32, 397-406. 
5. B.A. Korgel, S. Fullam, S. Connolly and D.J.Fitzmaurice Assembly and Self-Organization of Silver Nanocrystal Superlattices: Ordered "Soft Spheres" J.Phys. Chem. B 1998, 102, 8379- 8388.

6. M.P. Pileni. Nanocrystals self-assemblies: fabrication and collective properties.J. Phys. Chem., 2001, 105, 3358 - 3372

7. O.C. Compton and F.E. Osterloh. Evolution of Size and Shape in the Colloidal Crystallization of Gold Nanoparticles.J. Am. Chem. Soc., 2007, 129, 7793-7798.

8. M.P. Pileni. Acc. of Chem. Res., 2007, 40, 685-693.

9. A.I. Henry, A. Courty M.P. Pileni, P.A. Albouy and Israelachvili. Nano Lett. 2008, 8, 2000-2005.

10. M.I. Bodnarchuk, M.V. Kovalenko, H. Groiss, R. Resel, M. Reissner, G. Hesser, R.T. Lechner, W; Steiner, F. Schaeffler and W. Heiss. Small 2009, 5, 2247-2252.

11. P. Podsiadlo, B. Lee, V.B. Prakapenka, G.V. Krylova, R.D. Schaller, A. Demortière, and E.V Shevchenko. Nano Lett. 2011, 11, 579-588

12. S.M. Rupich, E.V. Shevchenko, M.I. Bodnarchuk, B. Lee, and D.V. Talapin. Size-Dependent Multiple Twinning in Nanocrystal Superlattices. J. Am. Chem. Soc., 2010, 132, 289-296.

13. J. Biais, L. Odberg and P. Stenius. Thermodynamic properties of microemulsions: Pseudophase equilibrium-Vapor pressure measurements J. Colloid Interface Sci. 1982, 86, 350-358.

14. M.P. Pileni. Self-assembly of inorganic nanocrystals: Fabrication and collective intrinsic properties. Acc. Chem Res., 2012, 45, 1965-1972

15. M. Bergström, E.V. Sturm, G. Salazar-Alvarez, H. Cölfen.. Mesocrystals in Biominerals and Colloidal Arrays. Acc. Chem. Res. 2015, 48, 1391-1402.

16. C. Wang, C. Siu, CJ. Zhang, and J. Fang. Understanding the Forces Acting in Self-Assembly and the Implications for Constructing Three-Dimensional (3D) Supercrystals.Nano Res. 2015, 8, 2445-2466.

17. M.A. Boles, M. Engel and D.V. Talapin, Self-Assembly of Colloidal Nanocrystals: From Intricate Structures to Functional Materials. Chem. Rev., 2016, 116, 11220-11289.

18. D. Haubold, A. Weiz, L. Borchardt, C. Ziegler, L. Bahrid, S. Kaskel, M. Ruck, and A. Eychmuller The Formation and Morphology of Nanoparticle Supracrystals Adv. Funct. Mat., 2016, 26, 4890

19. E. V.Shevchenko, D.V. Talapin, S. O'brien, C.B. Murray.. Polymorphism In $\mathrm{AB}_{13}$ Nanoparticle Superlattices: An Example Of Semiconductor-Metal Metamaterials J. Am. Chem. Soc. 2005, 127, 8741-8747.

20. E. V. Shevchenko, D.V. Talapin, N.A. Kotov. Structural diversity in binary nanoparticle superlattices. Nature 2006, 439, 55-58

21. Z. Yang, J. Wei, P. Bonville and M.P Pileni. Beyond Entropy: Magnetic Forces Induce Formation of Quasicrystalline Structure in Binary Nanocrystal Superlattices J. Am. Chem. Soc. 2015, 137, 4487-4493.

22. Z. Yang, N. Yang, J. Bergstromand M.P. Pileni Control of the oxygen and cobalt atoms diffusion through Co nanoparticles differing by their crystalline structure and size Adv. Funct. Mater. 2015, 25, 891-897

23. A. Taleb, C. Petit, and M.P. Pileni, M.P. Synthesis of highly monodisperse silver nanonarticles from AOT reverse micelles: a way to 2D and 3D self-organization Chem Materials, 1997, 9,950

24. A. Courty, A. Mermet, P.A. Albouy, E. Duval and M.P. Pileni, Self-organized Ag-nanocrystals in fcc "supra" crystals: Vibrational Coherence.Nature materials, 2005, 4, 395-398.

25. N. Zaitseva, D.Z. Rong, F.R. Leon, D. Krol. Optical Properties of CdSe Superlattices J. Am. Chem. Soc. 2005, 127, 10221-10226.

26. A.R. Tao, D.P. Ceperley, P. Sinsermsuksakul, A.R. Neureuther and P Yang, Self-Organized Silver Nanoparticles for Three-Dimensional Plasmonic Crystals.Nano Lett. 2008, 8, 4033-4038.

27. X. Ling, N. Schaeffer, S. Roland and M.P. Pileni. Superior Oxygen Stability of N-Heterocyclic Carbene-coated Au Nanocrystals Comparison with Dodecanethiol Langmuir, 2015, 31, 12873-12882

28. N. Yang, Z. Yang, M. Held, P. Bonville, P.A. Albouy, R. Lévy, and M.P. Pileni, Dispersion of Hydrophobic Co Supracrystal in Aqueous Solution ACS Nano, 2016,10, 2277-2286.

29. N. Yang, C. Deeb, J.L Pelouard, N. Felidj and M.P. Pileni, Water-Dispersed Hydrophobic Au Nanocrystal Assemblies with a Plasmon Fingerprint ACS Nano, 2017, 11, 7797-7806.

30. V. Germain and M.P. Pileni. Size distribution of cobalt nanocrystals: A key parameter in formation of columns and labyrinths in mesoscopic structure Adv. Mater., 2005, 17, 1424-1429

31. J. Wei, N. Schaeffer, P.A. Albouy, and M.P. Pileni Surface Plasmon Resonance Properties of Silver Nanocrystals Differing in Size and Coating Agent Ordered in 3D Supracrystals Chem. Mater. 2015, 27, 5614-5621

32. C. Allain and L Limat, Regular Patterns of Cracks Formed by Directional Drying of A Colloidal Suspension, Phys. Rev. Lett., 1995, 74, 2981-2984.

33. K.A. Shorlin, J.R. de Bruyn, M. Graham and S.W. Morris. Development and Geometry of Isotropic and Directional Shrinkage-Crack Patterns.Phys. Rev. E, 2000, 61, 6950-6957.

34. E.R. Dufresne, E. I. Corwin and N.A. Greenblatt. Flow and Fracture in Drying Nanoparticle Suspensions. Phys. Rev. Lett., 2003, 91, 224501

35. M.Heinrich, P. Gruber, S. Orso, U.A. Handge, and R. Spolenak. Dimensional Control of Brittle Nanoplatelets. A Statistical Analysis of a Thin Film Cracking Nano lett., 2006, 6, 2026-2030.

36. A. Groisman and E. Kaplan. An Experimental Study of Cracking Induced by Desiccation. Europhys. Lett., 1994, 25, 415-420.

37. T.S. Komatsu and S. Sasa. Pattern Selection of Cracks in Directionally Drying Fracture J. Appl. Phys., 1997, 36, 391-395.

38. K.T.Leung and Z. Néda. Pattern Formation and Selection in Quasistatic Fracture. Phys. Rev. Lett. 2000, 85, 662-665.

39. A.T. Ngo, J. Richardi and M.P. Pileni. Cracks in Magnetic Nanocrystal Films: Do Directional and Isotropic Crack Patterns Follow the Same Scaling Law? Nano Lett., 2008, 8, 2485-2489.

40. A.T. Ngo, J. Richardi and M.P. Pileni. Crack Patterns in Superlattices Made of Maghemite Nanocrystals. Phys. Chem. Chem. Phys., 2013, 15, 10666-10672.

41. I. Lisiecki, P. André, A. Filankembo, C. Petit, J. Tanori, T. Gulik-Krzywicki, B. W. Ninhamand M.P. Pileni Mesostructured Fluids. J. Phys. Chem. 1999, 103, 9176-8189 and 9168-9175

42. M. Kranenburg, M. Veturoli, and B. Smit. Phase Behavior and Induced Interdigitation in Bilayers Studied with Dissipative Particle Dynamics J. Phys. Chem. B., 2003, 107, 11491-11501 
43. J.F. Nagle and S. Tristram-Nagle. Structure of Lipid Bilayers Biochem. et Biophys. Acta - Reviews on Biomembranes 2000, 1469, 159-195.

44. J. Wei, C. Deeb, J.L. Pelouard, and M.P. Pileni Influence of Cracks on the Optical Properties of Silver Nanocrystals Supracrystal Films.ACS Nano, 2019, 13, 573-581

45. K.L. Young, M. B. Ross, M.G. Blaber, M. Rycenga, M.R. Jones, C. Zhang, A. J. Senesi, B. Lee,G.C. Schatz and C.A. Mirkin, Using DNA to Design Plasmonic Metamaterials with Tunable Optical Properties.Adv. Mater. 2014, 26, 653-659.

46. M.B. Ross, J.C. Ku, V.M, Vaccarezza, G.C Schatz, and C.A. Mirkin, Nat. Nanotech. 2015, 10, 453-458.

47. M.N. O’Brien, H.X. Lin, M. Girard, M.O. de la Cruz,. and C.A. Mirkin. Programming Colloidal Crystal Habit with Anisotropic Nanoparticle Building Blocks and DNA Bonds J. Am. Chem. Soc. 2016, 138, 14562-14565.

48. J.A. Mason, C.R. Laramy, C.T. Lai, M.N. O’Brien, Q.Y. Lin, V.P. Dravid, G.C. Schatz. and. C.A. Mirkin Contraction and Expansion of Stimuli-Responsive DNA Bonds in Flexible Colloidal Crystals J. Am. Chem. Soc. 2016, 138, 8722-8725.

49. D. J. Park, C. Zhang, J. C. Ku, Y. Zhou, G. C. Schatz and C.A. Mirkin Plasmonic photonic crystals realized through DNA-programmable assembly Proc. Natl. Acad. Sci. USA 2015, 112,977-981.

50. P. Aubertin, M.A. Ben Aissa, N. Raouafi, S. Joiret, A. Courty and E. Maisonhaute. Optical Response and Sers Properties of Individual Large Scale Supracrystals Made of Small Silver Nanocrystals.Nano Res. 2015, 8, 1615-1626.

51. D.E. Aspnes Local-Field Effects and Effective-Medium Theory: A Microscopic Perspective Am. J. Phys. 1982, 50, 704-709.

52. E. Duval, H. Portales, L. Saviot, M. Fujii, K. Sumitomo and S. Hayashi, Spatial Coherence Effect on the Low-FrequencyRaman Scattering from Metallic Nanoclusters Phys. Rev. B, 2001, 63, 075405

53. A. Mazzanti, Z. Yang, M.G. Silva, N. Yang, G. Rizza, P.E. Coulon, C. Manzoni, A.M. de Paula, G. Cerullo, G. Della Valle, G. and M.P. Pileni Light-heat conversion dynamics in highly diversified water-dispersed hydrophobic nanocrystal assemblies, Proc. Natl. Acad. Sci. USA, 2019, 116, 8161-8166.

54. I. Lisiecki, D. Polli, C. Yan, G. Soavi, E. Duval, G. Cerullo and M.P. Pileni. Coherent Longitudinal Acoustic Phonons in ThreeDimensional Supracrystals of Cobalt Nanocrystals Nano Lett., 2013, 13, 4914-4919

55. N. Goubet, C.Yan, D. Polli, H.Portalès, I.Arfaoui, G. Cerullo and M. P. Pileni Size and nanocrystallinity controlled gold nanocrystals: synthesis, electronic and mechanical properties Nano Lett., 2013, 13, 504-508.

56. M.G. Silva, et al. Universal saturation behavior in the transient optical response of plasmonic structures. Phys. Rev. B, 2018, 98, : 115407.

57. C.K. Sun, F. Vallée, L. Acioli E. Ippen and J.Fujimoto Femtosecond-tunable measurement of electron thermalization in gold Phys. Rev. B , 1994, 50, 15337-13342.

58 